\title{
KÄHLERIAN STRUCTURES AND D-HOMOTHETIC BI-WARPING
}

\author{
GHERICI BELDJILALI AND MOHAMED BELKHELFA
}

Communicated by Jean-Louis Clerc

\begin{abstract}
We introduce the notion of $\mathcal{D}$-homothetic bi-warping and starting from a Sasakian manifold $M$, we construct a family of Kählerian structures on the product $\mathbb{R} \times M$. After, we investigate conditions on the product of a cosymplectic or Kenmotsu manifold and the real line to be a family of conformal Kähler manifolds. We construct several examples.
\end{abstract}

MSC: 53C15, 53C25, 53C55, 53D25

Keywords: Kählerian structures, product manifolds, Sasakian structures

\section{Introduction}

To study manifolds with negative curvature, Bishop and O'Neill introduced the notion of warped product as a generalization of Riemannian product [1].

In 1985, using the warped product, Oubiña showed that there is a one-to-one correspondence between Sasakian and Kählerian structures [14].

Recently, building on the work of Tanno [16] (the homothetic deformation on contact metric manifold), Blair [6] introduced the notion of $\mathcal{D}$-homothetic warping. He used it for generating further results and examples of various structures. In particular, he showed in another way that there is a one-to-one correspondence between Sasakian and Kählerian structures.

Here by generalizing the $\mathcal{D}$-homothetic warping and following what made Blair in [6], we exceed this correspondence and we show that every Sasakian manifold $M$ generates a one-parameter family of Kählerian manifolds, thereby generalizing the results of Oubiña [14] and Blair [6]. On the other hand, we define a two-parameter family of conformally Kähler manifolds structures on the product manifold $\mathbb{I} \times M$ of an open interval and a cosymplectic or Kenmotsu manifold $M$ (Theorem 7), which is the first main result of the present paper. This text is organized in the following way.

Section 2 is devoted to the background of the structures which will be used in the sequel. 
In Section 3 we introduce the notion of $\mathcal{D}$-homothetic bi-warping and prove some basic properties.

Finally in Section 4 we give an application to some questions of the characterization of certain geometric structures with examples.

\section{Review Of Needed Notions}

An almost complex manifold with a Hermitian metric is called an almost Hermitian manifold. For an almost Hermitian manifold $(M, J, g)$ we thus have

$$
J^{2}=-1, \quad g(J X, J Y)=g(X, Y) .
$$

An almost complex structure $J$ is integrable, and hence the manifold is a complex manifold, if and only if its Nijenhuis tensor $N_{J}$ vanishes, with

$$
N_{J}(X, Y)=[J X, J Y]-[X, Y]-J[X, J Y]-J[J X, Y] .
$$

For an almost Hermitian manifold $(M, J, g)$, we define the fundamental Kähler form $\Omega$ as

$$
\Omega(X, Y)=g(X, J Y) .
$$

The triple $(M, J, g)$ is then called almost Kähler if $\Omega$ is closed, i.e., $\mathrm{d} \Omega=0$. An almost Kähler manifold with integrable $J$ is called a Kähler manifold, and thus is characterized by the conditions: $\mathrm{d} \Omega=0$ and $N_{J}=0$. One can prove that both these conditions combined are equivalent with the single condition

$$
\nabla J=0 .
$$

Definition 1 ([13]). A Hermitian manifold $(M, J, g)$ is called locally conformal Kähler (conformally Kähler) manifold if there exists a closed (exact) one-form $\theta$ (called the Lee form) such that

$$
\mathrm{d} \Omega=\theta \wedge \Omega .
$$

An odd-dimensional Riemannian manifold $\left(M^{2 n+1}, g\right)$ is said to be an almost contact metric manifold if there exist on $M$ a $(1,1)$ tensor field $\varphi$, a vector field $\xi$ (called the structure vector field) and a one-form $\eta$ such that

$$
\eta(\xi)=1, \quad \varphi^{2}(X)=-X+\eta(X) \xi, \quad g(\varphi X, \varphi Y)=g(X, Y)-\eta(X) \eta(Y)
$$

for any vector fields $X, Y$ on $M$. In particular, in an almost contact metric manifold we also have $\varphi \xi=0$ and $\eta \circ \varphi=0$. 
Such a manifold is said to be a contact metric manifold if $\mathrm{d} \eta=\Phi$, where $\Phi(X, Y)=$ $g(X, \varphi Y)$ is called the fundamental two-form of $M$.

On the other hand, the almost contact metric structure of $M$ is said to be normal if

$$
N_{\varphi}(X, Y)=[\varphi, \varphi](X, Y)+2 \mathrm{~d} \eta(X, Y) \xi=0
$$

for any $X, Y$, where $[\varphi, \varphi]$ denotes the Nijenhuis torsion of $\varphi$, given by

$$
[\varphi, \varphi](X, Y)=\varphi^{2}[X, Y]+[\varphi X, \varphi Y]-\varphi[\varphi X, Y]-\varphi[X, \varphi Y] .
$$

An almost contact metric structures $(\varphi, \xi, \eta, g)$ on $M$ is said to be

a) Sasaki $\Leftrightarrow \Phi=\mathrm{d} \eta$ and $(\varphi, \xi, \eta)$ is normal

b) Cosymplectic $\Leftrightarrow \mathrm{d} \Phi=\mathrm{d} \eta=0$ and $(\varphi, \xi, \eta)$ is normal

c) Kenmotsu $\Leftrightarrow \mathrm{d} \eta=0, \mathrm{~d} \Phi=2 \Phi \wedge \eta$ and $(\varphi, \xi, \eta)$ is normal

where $d$ denotes the exterior derivative. These manifolds can be characterized through their Levi-Civita connection, by requiring
1) Sasaki $\Leftrightarrow\left(\nabla_{X} \varphi\right) Y=g(X, Y) \xi-\eta(Y) X$
2) Cosymplectic $\Leftrightarrow \nabla \varphi=0$
3) Kenmotsu $\Leftrightarrow\left(\nabla_{X} \varphi\right) Y=g(\varphi X, Y) \xi-\eta(Y) \varphi X$

(see [2], [3], [9] and [17] ).

\section{D-Homothetic Bi-Warping}

Let $(M, \varphi, \xi, \eta, g)$ be an almost contact metric manifold with $\operatorname{dim} M=2 n+1$. The equation $\eta=0$ defines a $2 n$-dimensional distribution $\mathcal{D}$ on $M$. By an $2 n$ homothetic deformation or $\mathcal{D}$-homothetic deformation [16] we mean a change of structure tensors of the form

$$
\bar{\varphi}=\varphi, \quad \bar{\eta}=a \eta, \quad \bar{\xi}=\frac{1}{a} \xi, \quad \bar{g}=a g+a(a-1) \eta \otimes \eta
$$

where $a$ is a positive constant. If $(M, \varphi, \xi, \eta, g)$ is a contact metric structure with contact form $\eta$, then $(\bar{M}, \bar{\varphi}, \bar{\xi}, \bar{\eta}, \bar{g})$ is also a contact metric structure [16].

The idea works equally well for almost contact metric structures, the deformation

$$
\bar{\varphi}=\varphi, \quad \bar{\eta}=\lambda \eta, \quad \bar{\xi}=\frac{1}{\lambda} \xi, \quad \bar{g}=\alpha^{2} g+\beta^{2} \eta \otimes \eta
$$

is again an almost contact metric structure if $\lambda^{2}=\alpha^{2}+\beta^{2}$.

Putting $\alpha^{2}=a^{2}$ and $\beta^{2}=a^{2}\left(b^{2}-1\right)$ where $\lambda=a b \neq 0$, we get the deformation

$$
\bar{\varphi}=\varphi, \quad \bar{\eta}=a b \eta, \quad \bar{\xi}=\frac{1}{a b} \xi, \quad \bar{g}=a^{2} g+a^{2}\left(b^{2}-1\right) \eta \otimes \eta .
$$


Definition 2. Let $\left(M^{\prime}, g^{\prime}\right)$ be a Riemannian manifold and let $(M, \varphi, \xi, \eta, g)$ be an almost contact metric manifold, and $f, h$ be two smooth functions on $M^{\prime}$. The $\mathcal{D}$-homothetically bi-warped metric on $\tilde{M}=M^{\prime} \times M$ is defined by

$$
\tilde{g}=g^{\prime}+f^{2} g+f^{2}\left(h^{2}-1\right) \eta \otimes \eta
$$

where $f h \neq 0$ everywhere.

In particular, if $h= \pm 1$ then we have a warped product metric and if $h= \pm f$ we get the $\mathcal{D}$-homothetically warped metric [6].

Using the Koszul formula for the Levi-Civita connection of a Riemannian metric, one can obtain the following

Proposition 3. Let $\nabla^{\prime}, \nabla$ and $\tilde{\nabla}$ denote the Riemannian connections of $g^{\prime}, g$, and $\tilde{g}$ respectively. For all $X^{\prime}, Y^{\prime}$ vector fields on $M^{\prime}$ and independent of $M$ and $X, Y$ vector fields on $M$, we have the relations

$$
\begin{aligned}
\tilde{\nabla}_{X^{\prime}} Y^{\prime}= & \nabla_{X^{\prime}}^{\prime} Y^{\prime} \\
\tilde{g}\left(\tilde{\nabla}_{X^{\prime}} Y, Z\right)= & \tilde{g}\left(\tilde{\nabla}_{Y} X^{\prime}, Z\right)=-\tilde{g}\left(\tilde{\nabla}_{Y} Z, X^{\prime}\right) \\
= & f X^{\prime}(f) g(Y, Z)+f\left(\left(h^{2}-1\right) X^{\prime}(f)+f h X^{\prime}(h)\right) \eta(Y) \eta(Z) \\
\tilde{g}\left(\tilde{\nabla}_{X} Y, Z\right)= & \tilde{g}\left(\nabla_{X} Y, Z\right)+f^{2}\left(h^{2}-1\right)\left(\frac{1}{2}\left(g\left(\nabla_{X} \xi, Y\right)+g\left(\nabla_{Y} \xi, X\right)\right) \eta(Z)\right. \\
& +\mathrm{d} \eta(X, Z) \eta(Y)+\mathrm{d} \eta(Y, Z) \eta(X)) .
\end{aligned}
$$

Let $\sigma$ denotes the second fundamental form of $M$ in $M^{\prime} \times M$ and while $f, h$ are two functions on $M^{\prime}$, for emphasis we denote their gradients by $\operatorname{grad}^{\prime} f$ and $\operatorname{grad}^{\prime} h$ respectively. Then we have the following Theorem.

Theorem 4. For an almost contact metric manifold $\left(M^{2 n+1}, \varphi, \xi, \eta, g\right)$ and $a \mathcal{D}$ homothetically bi-warped metric on $\tilde{M}=M^{\prime} \times M$ we have the following assertions: 1) $M^{\prime}$ is a totally geodesic submanifold. 2) If $\operatorname{gard} f \operatorname{grad}^{\prime}(h-f)=0$ then $M$ is a quasi-umbilical submanifold and its second fundamental form is given by

$$
\sigma(X, Y)=-\frac{1}{2}\left(g(X, Y)+\left(h^{2}+f h-1\right) \eta(X) \eta(Y)\right) \operatorname{grad}^{\prime} f^{2} .
$$

3) The mean curvature vector of $M$ in $M^{\prime} \times M$ is

$$
\mathcal{H}=-\operatorname{grad}^{\prime}\left(\frac{\left(2 n+h^{2}\right) f^{2}}{2(2 n+1)}\right)
$$

4) $M$ is minimal if and only if

$$
h^{2}=\frac{c}{f^{2}}-2 n
$$


where $c>0$ in which case $M$ is quasi-umbilical and its second fundamental form is given by

$$
\sigma(X, Y)=\frac{1}{2}(g(X, Y)-(2 n+1) \eta(X) \eta(Y)) \operatorname{grad}^{\prime} f^{2}
$$

5) If $\mathrm{d} \eta(\xi, X)=0$ for every $X$ on $M$ (equivalently the integral curves of $\xi$ are geodesics), then the Reeb vector field $\xi$ is $\tilde{g}$-Killing if and only if it is $g$-Killing.

Proof: Recall that any submanifold $N$ in $\tilde{M}$ is a quasi-umbilical submanifold if its second fundamental form $\omega$ has the following form

$$
\omega(X, Y)=\alpha g(X, Y) \rho^{\prime}+\beta \eta(X) \eta(Y) \rho^{\prime}
$$

where $\alpha, \beta$ are two scalars, $X, Y$ are two vectors fields on $N$ and $\rho^{\prime}$ is a normal vectors field.

- If $\alpha=0$ then $N$ is cylindrical.

- If $\beta=0$ then $N$ is umbilical.

- If $\alpha=\beta=0$ then $N$ is geodesic.

1. Let $\sigma^{\prime}$ denotes the second fundamental form of $M^{\prime}$. Since we have $\tilde{\nabla}_{X^{\prime}} Y^{\prime}=$ $\nabla_{X^{\prime}}^{\prime} Y^{\prime}$ then

$$
\sigma^{\prime}=\tilde{\nabla}_{X^{\prime}} Y^{\prime}-\nabla_{X^{\prime}}^{\prime} Y^{\prime}=0
$$

2. From Proposition 3 we have

$$
\begin{aligned}
\tilde{g}\left(\tilde{\nabla}_{X} Y, Z^{\prime}\right)= & -f Z^{\prime}(f) g(X, Y)-f\left(\left(h^{2}-1\right) Z^{\prime}(f)+f h Z^{\prime}(h)\right) \eta(X) \eta(Y) \\
= & -f g^{\prime}\left(g(X, Y) \operatorname{grad}^{\prime} f+\left(\left(h^{2}-1\right) \operatorname{grad}^{\prime} f\right.\right. \\
& \left.\left.+f h \operatorname{grad}^{\prime} h\right) \eta(X) \eta(Y), Z^{\prime}\right)
\end{aligned}
$$

since $\tilde{g}\left(\nabla_{X} Y, Z^{\prime}\right)=0$ and knowing that $\sigma=\tilde{\nabla}_{X} Y-\nabla_{X} Y$ one ends with $\sigma(X, Y)=-\frac{1}{2} g(X, Y) \operatorname{grad}^{\prime} f^{2}-\frac{1}{2}\left(\left(h^{2}-1\right) \operatorname{grad}^{\prime} f^{2}+f^{2} \operatorname{grad}^{\prime} h^{2}\right) \eta(X) \eta(Y) . \quad(*)$

If $\operatorname{grad}^{\prime} h=\operatorname{grad}^{\prime} f$ then we obtain

$$
\sigma(X, Y)=-f\left(g(X, Y)+\left(h^{2}+f h-1\right) \eta(X) \eta(Y)\right) \operatorname{grad}^{\prime} f .
$$


3. Knowing that the mean curvature vector of $M$ in $M^{\prime} \times M$ is given by

$$
\mathcal{H}=\frac{1}{2 n+1} \operatorname{tr}_{g} \sigma=\frac{1}{2 n+1} \sum_{i=1}^{2 n+1} \sigma\left(e_{i}, e_{i}\right)
$$

where $\left\{e_{i}\right\}_{i=1,2 n+1}$ is an orthonormal basis on $M$ so

$$
\begin{aligned}
\mathcal{H} & =\frac{1}{2 n+1} \sum_{i=1}^{i=2 n+1} \sigma\left(e_{i}, e_{i}\right) \\
& =-\frac{f}{2 n+1} \sum_{i=1}^{i=2 n+1}\left((2 n+1) \operatorname{grad}^{\prime} f+\left(\left(h^{2}-1\right) \operatorname{grad}^{\prime} f+f h \operatorname{grad}^{\prime} h\right)\right) \\
& =-\frac{1}{2(2 n+1)} \operatorname{grad}^{\prime}\left(\left(2 n+h^{2}\right) f^{2}\right)
\end{aligned}
$$

4. The submanifold $M$ is minimal, i.e., the mean curvature $\mathcal{H}$ is zero, using the result (3), we get

$$
h^{2}=\frac{c}{f^{2}}-2 n
$$

Now replacing $\operatorname{grad}^{\prime} h^{2}=-\frac{c}{f^{4}} \operatorname{grad}^{\prime} f^{2}$ in $(*)$ we find

$$
\sigma(X, Y)=\frac{1}{2}(g(X, Y)-(2 n+1) \eta(X) \eta(Y)) \operatorname{grad}^{\prime} f^{2} .
$$

5. For every two vectors fields $\tilde{X}=X^{\prime}+X$ and $\tilde{Y}=Y^{\prime}+Y$ on $\tilde{M}$ we have that

$$
\xi \quad \text { is } \quad \tilde{g}-\operatorname{Killing} \Leftrightarrow \tilde{g}\left(\tilde{\nabla}_{\tilde{X}} \xi, \tilde{Y}\right)+\tilde{g}\left(\tilde{\nabla}_{\tilde{Y}} \xi, \tilde{X}\right)=0
$$

and

$$
\begin{aligned}
\tilde{g}\left(\tilde{\nabla}_{\tilde{X}} \xi, \tilde{Y}\right)+\tilde{g}\left(\tilde{\nabla}_{\tilde{Y}} \xi, \tilde{X}\right)= & \tilde{g}\left(\tilde{\nabla}_{X^{\prime}+X} \xi, Y^{\prime}+Y\right)+\tilde{g}\left(\tilde{\nabla}_{Y^{\prime}+Y} \xi, X^{\prime}+X\right) \\
= & \tilde{g}\left(\tilde{\nabla}_{X^{\prime}} \xi, Y\right)+\tilde{g}\left(\tilde{\nabla}_{X} \xi, Y^{\prime}\right)+\tilde{g}\left(\tilde{\nabla}_{X} \xi, Y\right) \\
& +\tilde{g}\left(\tilde{\nabla}_{Y^{\prime}} \xi, X\right)+\tilde{g}\left(\tilde{\nabla}_{Y} \xi, X^{\prime}\right)+\tilde{g}\left(\tilde{\nabla}_{Y} \xi, X\right) .
\end{aligned}
$$

Suppose $\mathrm{d} \eta(\xi, X)=0$, i.e., $\xi \eta(X)=\eta\left(\nabla_{\xi} X\right)$ then, we can easily check the following assertions

$$
\tilde{g}\left(\tilde{\nabla}_{X^{\prime}} \xi, Y\right)=\frac{1}{2} X^{\prime}\left(f^{2} h^{2}\right) \eta(Y)
$$




$$
\begin{gathered}
\tilde{g}\left(\tilde{\nabla}_{X} \xi, Y^{\prime}\right)=-\frac{1}{2} Y^{\prime}\left(f^{2} h^{2}\right) \eta(X) \\
\tilde{g}\left(\tilde{\nabla}_{X} \xi, Y\right)=\tilde{g}\left(\nabla_{X} \xi, Y\right)+f^{2}\left(h^{2}-1\right) \mathrm{d} \eta(X, Y) .
\end{gathered}
$$

When replacing in $(* *)$ we obtain also

$$
\begin{aligned}
\tilde{g}\left(\tilde{\nabla}_{\tilde{X}} \xi, \tilde{Y}\right)+\tilde{g}\left(\tilde{\nabla}_{\tilde{Y}} \xi, \tilde{X}\right) & =\tilde{g}\left(\nabla_{X} \xi, Y\right)+\tilde{g}\left(\nabla_{Y} \xi, X\right) \\
& =f^{2}\left(g\left(\nabla_{X} \xi, Y\right)+g\left(\nabla_{Y} \xi, X\right)\right) .
\end{aligned}
$$

This completes the proof.

\section{Application to Geometric Structures}

In the remaining part of the paper, we consider the case where $M^{\prime}=\mathbb{R}, M$ is an almost contact metric manifolds and the metric

$$
\tilde{g}=\mathrm{d} t^{2}+f^{2} g+f^{2}\left(h^{2}-1\right) \eta \otimes \eta
$$

where $f, h$ are functions on $\mathbb{R}$. For brevity we denote the unit tangent field to $\mathbb{R}$ by $\partial_{t}$. In this case the proposition (3) becomes

Proposition 5. Let $(M, \varphi, \xi, \eta, g)$ be an almost contact metric manifold. Let $\nabla$ and $\tilde{\nabla}$ denote the Riemannian connections of $g$, and $\tilde{g}$ respectively. For all $X, Y$ vector fields tangent to $M$ and independent of $\mathbb{R}$, we have

$$
\begin{gathered}
\tilde{g}\left(\tilde{\nabla}_{\partial_{t}} Y, Z\right)=\tilde{g}\left(\tilde{\nabla}_{Y} \partial_{t}, Z\right)=-\tilde{g}\left(\tilde{\nabla}_{Y} Z, \partial_{t}\right)=f f^{\prime} g(\varphi Y, \varphi Z)+f h(f h)^{\prime} \eta(Y) \eta(Z) \\
\tilde{g}\left(\tilde{\nabla}_{X} Y, Z\right)=\tilde{g}\left(\nabla_{X} Y, Z\right)+f^{2}\left(h^{2}-1\right)\left(\frac{1}{2}\left(g\left(\nabla_{X} \xi, Y\right)+g\left(\nabla_{Y} \xi, X\right)\right) \eta(Z)\right. \\
+\mathrm{d} \eta(X, Z) \eta(Y)+\mathrm{d} \eta(Y, Z) \eta(X)) .
\end{gathered}
$$

Next, we introduce a class of almost complex structure $\tilde{J}$ on manifold $\tilde{M}$

$$
\tilde{J}\left(\partial_{t}, X\right)=\left(f h \eta(X) \partial_{t}, \varphi X-\frac{1}{f h} \xi\right)
$$

for any vector filds $X$ of $M$ where $f, h$, are functions on $\mathbb{R}$ and $f h \neq 0$ everywhere. That $J^{2}=-I$ is easily checked and for all $\tilde{X}=\left(a \partial_{t}, X\right), \tilde{Y}=\left(b \partial_{t}, Y\right)$ on $\tilde{M}$ we can see that $\tilde{g}$ is almost Hermitian with respect to $\tilde{J}$, i.e.,

$$
\tilde{g}(\tilde{J} \tilde{X}, \tilde{J} \tilde{Y})=\tilde{g}(\tilde{X}, \tilde{Y}) .
$$


On the other hand, the fundamental two-form $\tilde{\Omega}$ of $(\tilde{J}, \tilde{g})$ is

$$
\tilde{\Omega}\left(\left(a \frac{\partial}{\partial t}, X\right),\left(b \frac{\partial}{\partial t}, Y\right)\right)=\tilde{g}\left(\left(a \frac{\partial}{\partial t}, X\right), \tilde{J}\left(b \frac{\partial}{\partial t}, Y\right)\right)
$$

we can check that is very simply as follows

$$
\tilde{\Omega}=f(2 h \mathrm{~d} t \wedge \eta+f \Phi)
$$

we have immediately that

$$
\mathrm{d} \tilde{\Omega}=f\left(-2 h \mathrm{~d} t \wedge \mathrm{d} \eta+2 f^{\prime} \mathrm{d} t \wedge \Phi+f \mathrm{~d} \Phi\right) .
$$

For the special cases we have the following

$$
\begin{aligned}
& \text { 1) contact metric } \mathrm{d} \tilde{\Omega}=-2 f\left(h-f^{\prime}\right) \mathrm{d} t \wedge \Phi \\
& \text { 2) almost cosymplectic } \mathrm{d} \tilde{\Omega}=2 f f^{\prime} \mathrm{d} t \wedge \Phi \\
& \text { 3) almost Kenmotsu } \mathrm{d} \tilde{\Omega}=2 f\left(f^{\prime} \mathrm{d} t+f \eta\right) \wedge \Phi .
\end{aligned}
$$

We note that $\tilde{\Omega}$ is closed in the contact metric case if and only if $h=f^{\prime}$ and in the almost cosymplectic case if and only if $f$ is constant. In the Kenmotsu case cannot be closed; it would force $f$ to be zero.

Now, putting $h=f^{\prime}$, the structure $(\tilde{g}, \tilde{J})$ (see (5), (6)) becomes

$$
\begin{aligned}
\tilde{g} & =\mathrm{d} t^{2}+f^{2} g+f^{2}\left(f^{\prime 2}-1\right) \eta \otimes \eta \\
\tilde{J}\left(a \frac{\partial}{\partial t}, X\right) & =\left(f f^{\prime} \eta(X) \frac{\partial}{\partial t}, \varphi X-\frac{a}{f f^{\prime}} \xi\right)
\end{aligned}
$$

where $f f^{\prime} \neq 0$ on $M$ everywhere, and $X$ any vector field of $M$.

We denote by $N_{\tilde{J}}$ the Nijenhuis tensor of the almost complex structure $\tilde{J}$. Then from (11) we have

$$
\begin{aligned}
N_{\tilde{J}}((0, X),(0, Y)) & =\left(f f^{\prime} N_{\varphi}^{(2)}(X, Y) \frac{\partial}{\partial t}, N_{\varphi}^{(1)}(X, Y)\right) \\
N_{\tilde{J}}\left(\left(\frac{\partial}{\partial t}, 0\right),(0, X)\right) & =\left(N_{\varphi}^{(4)}(X) \frac{\partial}{\partial t}, \frac{1}{f f^{\prime}} N_{\varphi}^{(3)}(X)\right)
\end{aligned}
$$

for any vector fields $X, Y$ of $M$. We denote by $N_{\varphi}^{(1)}, N_{\varphi}^{(2)}, N_{\varphi}^{(3)}$ and $N_{\varphi}^{(4)}$ the following tensor fields on $M$ defined respectively by

$$
\begin{aligned}
N_{\varphi}^{(1)}(X, Y) & =[\varphi, \varphi](X, Y)+2 \mathrm{~d} \eta(X, Y) \xi \\
N_{\varphi}^{(2)}(X) & =\left(L_{\varphi X}\right)(Y)-\left(L_{\varphi_{Y}}\right)(X) \\
N_{\varphi}^{(3)}(X) & =-\left(L_{\xi} \varphi\right)(X), \quad N_{\varphi}^{(4)}(X)=\left(L_{\xi} \eta\right)(X) .
\end{aligned}
$$


Proposition 6 ([3]). For an almost contact manifold $M=(M, \varphi, \xi, \eta)$ the vanishing of the tensor field $N_{\varphi}^{(1)}$ implies the vanishing of the tensor fields $N_{\varphi}^{(2)}, N_{\varphi}^{(3)}$ and $N_{\varphi}^{(4)}$.

From the above proposition, we see that an almost contact metric manifold $M=$ $(M, \varphi, \xi, \eta)$ is normal if and only if $N_{\varphi}^{(1)}$ vanishes everywhere on $M$ ([3], p.81).

Therefore, summing up the arguments above, we have the following main theorem

Theorem 7. 1. The almost contact metric structure on $M$ is a contact metric structure if and only if the almost Hermitian structure $(\tilde{g}, \tilde{J})$ is almost Kähler (i.e., $\mathrm{d} \tilde{\Omega}=0$ ) for all function $f$ on $\mathbb{R}$ such that $f f^{\prime} \neq 0$. In addition, the structure on $M$ is Sasakian if and only if the structure $(\tilde{g}, \tilde{J})$ on $\tilde{M}$ is Kählerian.

2. The almost contact metric structure on $M$ is almost cosymplectic if and only if the almost Hermitian structure $(\tilde{g}, \tilde{J})$ satisfies $\mathrm{d} \tilde{\Omega}=2 f f^{\prime}(\mathrm{d} t \wedge \Phi)$ in which case the structure is conformally almost Kähler. In addition, the structure on $M$ is cosymplectic if and only if the structure $(\tilde{g}, \tilde{J})$ on $\tilde{M}$ is conformally Kähler.

3. The almost contact metric structure on $M$ is almost Kenmotsu if and only if the almost Hermitian structure $(\tilde{g}, \tilde{J})$ satisfies $\mathrm{d} \tilde{\Omega}=2 f\left(f^{\prime} \mathrm{d} t+f \eta\right) \wedge \Phi$ in which case the structure is conformally almost Kähler if and only if $\eta$ is exact. In addition, if the structure on $M$ is Kenmotsu then the structure $(\tilde{g}, \tilde{J})$ on $\tilde{M}$ is conformally Kähler if and only if $\eta$ is exact. Moreover, if $\eta=-\mathrm{d} \beta$ for some $\beta \in C^{\infty}(\tilde{M})$ then $\mathrm{e}^{2(\beta-\ln |f|)} \tilde{g}$ will be a Kähler metric on $\tilde{M}$.

Proof: The necessity was observed above for both cases (see (3)). For the sufficiency, first observe that from equation (8) where $h=f^{\prime}$ we have

$$
\mathrm{d} \tilde{\Omega}\left(\left(\frac{\partial}{\partial t}, 0\right),(0, X),(0, Y)\right)=2 f f^{\prime}(\Phi-\mathrm{d} \eta)(X, Y) .
$$

If $\mathrm{d} \tilde{\Omega}=0$, then the equation (12) gives $\Phi=\mathrm{d} \eta$ and we have a contact metric structure.

So, if $M$ is Sasakian then the structure $(g, J)$ is Kählerian.

2) If $\mathrm{d} \tilde{\Omega}=2 f f^{\prime}(\mathrm{d} t \wedge \Phi)$, then the equation (12) gives $\mathrm{d} \eta=0$ and applying $\mathrm{d}$ to $\mathrm{d} \tilde{\Omega}=2 f f^{\prime}(\mathrm{d} t \wedge \Phi)$ we have $\mathrm{d} \Phi=0$ and hence an almost cosymplectic structure on $M$. 
Now consider the metric $\bar{g}=\frac{1}{f^{2}} \tilde{g}$, it is almost Hermitian with respect to $\tilde{J}$ and its fundamental two-form $\bar{\Omega}=\frac{1}{f^{2}} \tilde{\Omega}$. Then

$$
\begin{aligned}
\mathrm{d} \bar{\Omega} & =\frac{-2 f^{\prime}}{f^{3}} \mathrm{~d} t \wedge \tilde{\Omega}+\frac{1}{f^{2}} \mathrm{~d} \tilde{\Omega} \\
& =\frac{-2 f^{\prime}}{f^{3}} \mathrm{~d} t \wedge f\left(2 f^{\prime} \mathrm{d} t \wedge \eta+f \Phi\right)+\frac{1}{f}\left(-2 f^{\prime} \mathrm{d} t \wedge \mathrm{d} \eta+2 f^{\prime} \mathrm{d} t \wedge \Phi+f \mathrm{~d} \Phi\right) \\
& =0
\end{aligned}
$$

giving a conformally almost Kähler structure.

3) If $\mathrm{d} \tilde{\Omega}=2 f\left(f^{\prime} \mathrm{d} t+f \eta\right) \wedge \Phi$, then the equation (12) gives $\mathrm{d} \eta=0$ and applying $\mathrm{d}$ to $\mathrm{d} \tilde{\Omega}=2 f\left(f^{\prime} \mathrm{d} t+f \eta\right) \wedge \Phi$ we get

$$
\left(f^{\prime} \mathrm{d} t+f \eta\right) \wedge \mathrm{d} \Phi=2 f^{\prime} \mathrm{d} t \wedge \eta \wedge \Phi
$$

so that $\mathrm{d} \Phi=2 \eta \wedge \Phi$, and hence an almost Kenmotsu structure on $M$.

Using (7) and (9,3) with $h=f^{\prime}$ we get

$$
\mathrm{d} \tilde{\Omega}=2(\mathrm{~d}(\ln |f|)+\eta) \wedge \tilde{\Omega} .
$$

From definition (1), it is obvious that $\tilde{M}$ is conformally Kähler if and only if $\eta$ is exact. Now, consider the metric $\widehat{g}=\mathrm{e}^{2(\beta-\ln |f|)} \tilde{g}$ with $\beta \in C^{\infty}(\tilde{M})$. This metric is Hermitian with respect to $\tilde{J}$ and its fundamental two-form $\widehat{\Omega}=\mathrm{e}^{2(\beta-\ln |f|)} \tilde{\Omega}$. Then, by straightforward calculations, using (13) and $\eta=-\mathrm{d} \beta$ we obtain $\mathrm{d} \widehat{\Omega}=0$ and this completes the proof.

\section{Special cases:}

- For $f=t$ with $h=f^{\prime}$, where $t>0$ and by (10) we get the metric cone (see [14])

$$
\tilde{g}=\mathrm{d} t^{2}+t^{2} g, \quad \tilde{J}\left(a \frac{\partial}{\partial t}, X\right)=\left(t \eta(X) \frac{\partial}{\partial t}, \varphi X-\frac{a}{t} \xi\right) .
$$

- For $f=h=e^{t}$, and by (10) we get the $\mathcal{D}$-homothetic warping (see [6])

$$
\begin{aligned}
\tilde{g} & =\mathrm{d} t^{2}+\mathrm{e}^{2 t} g+\mathrm{e}^{2 t}\left(\mathrm{e}^{2 t}-1\right) \eta \otimes \eta \\
\tilde{J}\left(a \frac{\partial}{\partial t}, X\right) & =\left(\mathrm{e}^{2 t} \eta(X) \frac{\partial}{\partial t}, \varphi X-a \mathrm{e}^{-2 t} \xi\right) .
\end{aligned}
$$

Example 8. We denote the Cartesian coordinates in a three-dimensional Euclidean space $E^{3}$ by $(x, y, z)$ and define a symmetric tensor field $g$ by

$$
g=\left(\begin{array}{ccc}
\rho^{2}+\tau^{2} & 0 & -\tau \\
0 & \rho^{2} & 0 \\
-\tau & 0 & 1
\end{array}\right)
$$


where $\rho$ and $\tau$ are functions on $E^{3}$ such that $\rho \neq 0$ everywhere.

Further, we define an almost contact metric $(\varphi, \xi, \eta)$ on $E^{3}$ by

$$
\varphi=\left(\begin{array}{ccc}
0 & -1 & 0 \\
1 & 0 & 0 \\
0 & -\tau & 0
\end{array}\right), \quad \xi=\left(\begin{array}{l}
0 \\
0 \\
1
\end{array}\right), \quad \eta=(-\tau, 0,1) .
$$

The fundamental one-form $\eta$ and the two-form $\Phi$ have the forms

$$
\eta=\mathrm{d} z-\tau \mathrm{d} x \quad \text { and } \quad \Phi=-2 \rho^{2} \mathrm{~d} x \wedge \mathrm{d} y
$$

and hence

$$
\mathrm{d} \eta=\tau_{2} \mathrm{~d} x \wedge \mathrm{d} y+\tau_{3} \mathrm{~d} x \wedge \mathrm{d} z, \quad \mathrm{~d} \Phi=-4 \rho_{3} \rho \mathrm{d} x \wedge \mathrm{d} y \wedge \mathrm{d} z
$$

where $\rho_{i}=\frac{\partial \rho}{\partial x_{i}}$ and $\tau_{i}=\frac{\partial \tau}{\partial x_{i}}$.

Knowing that the components of the Nijenhuis tensor $N_{\varphi}$ in (2) can be written as

$$
N_{k j}^{i}=\varphi_{k}^{l}\left(\partial_{l} \varphi_{j}^{i}-\partial_{j} \varphi_{l}^{i}\right)-\varphi_{j}^{l}\left(\partial_{l} \varphi_{k}^{i}-\partial_{k} \varphi_{l}^{i}\right)+\eta_{k}\left(\partial_{j} \xi^{i}\right)-\eta_{j}\left(\partial_{k} \xi^{i}\right)
$$

where the indices $i, j, k$ and $l$ run over the range $1,2,3$, then by a direct computation we can verify that

$$
N_{k j}^{i}=0, \quad \text { for all } \quad i, j, k
$$

implying that the structure $(\varphi, \xi, \eta, g)$ is normal. From definitions in (3), the structure $(\varphi, \xi, \eta, g)$ is a

1) Sasaki, when $\tau_{2}=-2 \rho^{2}$ and $\tau_{3}=0$

2) cosymplectic, when $\rho_{3}=0, \tau_{2}=0$ and $\tau_{3}=0$

3) Kenmotsu, when $\rho_{3}=\rho, \tau_{2}=0$ and $\tau_{3}=0$.

Using the above cases and Theorem (7), the manifold $\left(\mathbb{R} \times E^{3}, \tilde{g}, \tilde{J}\right)$ is

1) Kählerian, when $\tau_{2}=-2 \rho^{2}$ and $\tau_{3}=0$

2) conformally Kählerian, when $\rho_{3}=0, \tau_{2}=0$ and $\tau_{3}=0$

3) conformally Kählerian, when $\rho_{3}=\rho, \tau_{2}=0$ and $\tau_{3}=0$.

Note that

$$
\tilde{g}=\left(\begin{array}{cccc}
1 & 0 & 0 & 0 \\
0 & f^{2}\left(\rho^{2}+f^{\prime 2} \tau^{2}\right) & 0 & -\tau f^{2} f^{\prime 2} \\
0 & 0 & f^{2} \rho^{2} & 0 \\
0 & -\tau f^{2} f^{\prime 2} & 0 & f^{2} f^{\prime 2}
\end{array}\right), \quad \tilde{J}=\left(\begin{array}{cccc}
0 & -\tau f f^{\prime} & 0 & f f^{\prime} \\
0 & 0 & -1 & 0 \\
0 & 1 & 0 & 0 \\
-\frac{1}{f f^{\prime}} & 0 & -\tau & 0
\end{array}\right) .
$$




\section{Acknowledgements}

The first author wishes to thank Professor Aissa Wade for her hospitality, kindness and helpful suggestions during his visit in May 2015 to Penn State University, USA.

\section{References}

[1] Bishop R. and O’Neill B., Manifolds of Negative Curvature, Trans. Amer. Math. Soc. 46 (1969) 1-49.

[2] Blaga A., Geometrical Aspects of Paracontact Pair Structures, J. Geom. Symmetry Phys. 34 (2014) 1-12.

[3] Blair D., Riemannian Geometry of Contact and Symplectic Manifolds, $2^{\text {nd }}$ Edn, Progress in Mathematics, Birhauser, Boston 2002.

[4] Blair D. and Oubiña J., Conformal and Related Changes of Metric on the Product of Two Almost Contact Metric Manifolds, Publ. Mat. 234 (1990) 199-207.

[5] Blair D. and Showers D., Almost Contact Manifolds With Killing Structures Tensors II, J. Diff. Geom. 9 (1974) 577-582.

[6] Blair D., D-Homothetic Warping and Appolications to Geometric Structures and Cosmology, African Diaspora Journal of Math. 14 (2013) 134-144.

[7] Boyer C., Galicki K. and Matzeu P., On Eta-Einstein Sasakian Geometry, Comm. Math. Phys. 262 (2006) 177-208.

[8] Calabi E. and Eckmann B., A Class of Compact Complex Manifolds which are not Algebraic, Ann. Math. 58 (1953) 494-500.

[9] Kenmotsu K., A Class of Almost Contact Riemannian Manifolds, J. Tohoku Math. 24 (1972) 93-103.

[10] Marrero J., The Local Structure of Trans-Sasakian Manifolds, Annali di Matematica Pura ed Applicata 162 (1992) 77-86.

[11] Morimoto A., On Normal Almost Contact Structures, J. Math. Soc. Japan 15 (1963) 420-436.

[12] Munteanu M., A Note on Doubly Warped Product Contact CR-Submanifolds in Trans-Sasakian Manifolds, Acta Math. Hungar. 116 (2007) 121-126.

[13] Ornea L. and Verbitsky M., Locally Conformally Kaehler Manifolds with Potential, Mathematische Annalen 148 (2010) 25-33.

[14] Oubiña J. A., New Classes of Almost Contact Metric Structures, Publ. Math. Debrecen 32 (1985) 187-193. 
[15] Sparks J., Sasaki-Einstein Manifolds, Surveys Diff. Geom. 16 (2011) 265324.

[16] Tanno S., The Topology of Contact Riemannian Manifolds, Illinois J. Math. 12 (1968) 700-717.

[17] Yano K. and Kon M., Structures on Manifolds, World Scientific, Singapore 1984.

Gherici Beldjilali

Laboratoire de Physique Quantique de la Matière et de Modélisation Mathématique (LPQ3M)

Université de Mascara, BP 305

Route de Mamounia 29000, Mascara

ALGERIA

E-mail address: Beldjilali29@gmail.com

Mohamed Belkhelfa

TAIBAH University

Department of Mathematics

College of Science and Arts, Al Ula

Kingdom of Saudi Arabia

E-mail address: mohamed.belkhelfa@gmail.com 